\title{
Rapid estimation of protein, lipid, and dry weight in microalgae using a portable LED fluorometer
}

\author{
Nathan G. Schoepp a,d,1 William S. Ansari b,d,1 Jason A. Dallwig ${ }^{\text {c }}$, Debra Gale ${ }^{\text {c }}$, \\ Michael D. Burkart ${ }^{\mathrm{a}, \mathrm{d}}$, Stephen P. Mayfield ${ }^{\mathrm{b}, \mathrm{d}, *}$ \\ a Department of Chemistry \& Biochemistry, University of California San Diego, 9500 Gilman Drive, La Jolla, CA 92093, United States \\ b Division of Biological Sciences, University of California San Diego, 9500 Gilman Drive MC0368, La Jolla, CA 92093, United States \\ c Thermo Fisher Scientific, 29851 Willow Creek Road, Eugene, OR 97402, United States \\ d The California Center for Algae Biotechnology, University of California San Diego, 9500 Gilman Drive MC0368, La Jolla, CA 92093, United States
}

\section{A R T I C L E I N F O}

\section{Article history:}

Received 13 March 2015

Received in revised form 3 June 2015

Accepted 16 June 2015

Available online 24 June 2015

\section{Keywords:}

Protein estimation

Lipid content

Dry weight

Portable

Fluorometer

\begin{abstract}
A B S T R A C T
As the industry surrounding microalgae continues to develop, there is a growing need for reliable, ready-to-use technologies for measuring the growth and composition of algal cultures. These can be new technologies or adapted existing technologies presently used for similar applications in other systems. Here we demonstrate the use of an LED fluorometer for the rapid estimation of dry weight, protein, and lipid content from two strains of microalgae. The instrument was successfully used to determine the dry weight of Chlamydomonas reinhardtii (CC-3491) and Scenedesmus dimorphus (UTEX 1237) cultures, at densities up to $1.58 \mathrm{~g} / \mathrm{L}$. Soluble protein was also measured using the instrument, and was highly comparable (average within $3 \%$ ) to results obtained using both the Bradford and Lowry methods. Lastly, neutral lipid accumulation induced by nitrogen starvation was estimated via BODIPY 495/505 fluorescence. The basic methods developed here can easily be applied to any strain of microalgae or cyanobacteria, and demonstrate reliable, cost-effective, single-instrument methods for the determination of several key parameters in the cultivation of photosynthetic microorganisms.
\end{abstract}

(c) 2015 Published by Elsevier B.V.

\section{Introduction}

Microalgae's potential as a bio-product crop rests on its areal productivity and an ability to produce commercially relevant products. Accurate determination of productivity and biomass composition is key for both research and industrial production. Established methods for the measurement of biomass, protein, and lipid content are reliable and accurate, but are involved processes not well suited to portability due to the complex and expensive equipment required [1-3]. However, several analytical techniques and the associated instrumentation have recently seen large improvements in both portability and cost with little sacrifice in accuracy [4-6]. In the emerging field of algae biotechnology, portability and cost are two highly desired characteristics of any technique or instrument. Low-cost options for culture monitoring will become increasingly important as industries develop around microalgae, especially those attempting to compete with inexpensive commodity markets such as fuel and animal feed. It is with this scenario in mind that we tested and developed protocols for biomass, protein,

\footnotetext{
* Corresponding author at: 9500 Gilman Drive, Bonner Hall 2150C, La Jolla, CA 92093 United States.

E-mail address: smayfield@ucsd.edu (S.P. Mayfield).

1 Both authors contributed equally to this work.
}

and lipid determination using an inexpensive and commercially available LED fluorometer.

The assays described were developed using the Qubit 2.0, a portable LED fluorometer equipped with two LEDs: blue with a maximum of $\sim 470 \mathrm{~nm}$, and red with a maximum of $\sim 635 \mathrm{~nm}$ (filters 430 $495 \mathrm{~nm}$ and 600-645 nm, respectively). Two emission filters from $510-580 \mathrm{~nm}$ and $665-725 \mathrm{~nm}$ are built into the instrument. These optics make the instrument well suited for measuring chlorophyll fluorescence in microalgae without any modification, enabling the use of chlorophyll $a$ fluorescence as a corollary for other parameters such as dry weight or culture density. Additionally, the instrument is spectrally compatible with lipophilic BODIPY dyes, and hence well suited for estimation of total lipid content. BODIPY fluorophores have long been used with fluorometers for total lipid determination, and their potential use expands well beyond this purpose $[7,8]$. In addition to lipid, a protein assay has been optimized for protein concentrations of $12.5 \mu \mathrm{g} / \mathrm{mL}-5 \mathrm{mg} / \mathrm{mL}$ and can tolerate between $1-20 \mu \mathrm{L}$ of sample. The assay utilizes a solvatochromatic dye that exhibits a large increase in fluorescence signal in the presence of a protein-detergent complex. Several typical issues often encountered in protein assays, such as interference by salts and cellular contents such as DNA, free amino acids, and various solvents, are well-tolerated, and have been used to determine purified algal protein concentrations previously [9]. 
Microalgae continue to grow as a production platform for biofuels, therapeutic proteins, and high-value products such as omega- 3 oils and vitamins $[10,11]$. The two strains tested here represent model laboratory and production strains. Chlamydomonas reinhardtii has long served as a genetic and metabolomics model alga, and a photosynthetic bio-factory for recombinant proteins [12,13]. Outside of the laboratory, Scenedesmus dimorphus has proven an immensely promising bioproducts strain due to its high tolerance of both abiotic and biotic stresses [14]. Scenedesmus sp. have recently been genetically manipulated, adding to the likelihood that the species will become an important producer in the emerging industry [15]. The ability of the fluorometerbased assays to work with both these species of microalgae, along with their relative speed demonstrates the potential of these assays to be versatile tools in the field of microalgae research.

\section{Materials and methods}

\subsection{Maintenance of stock cultures}

C. reinhardtii ( $\mathrm{Cr}$ ) (CC-3491) was obtained from the Chlamydomonas Resource Center, University of Minnesota, USA. S. dimoprhus (Sd) (UTEX 1237) was obtained from the University of Texas at Austin Culture Collection, Texas, USA. Strains were maintained on 1.5\% agar plates of Sueoka's High Salt Medium (HSM) [16]. Stocks were maintained in ambient conditions at $24{ }^{\circ} \mathrm{C}$ under $6500 \mathrm{~K}$ fluorescent lights at a constant irradiance of $60 \mu \mathrm{mol} \mathrm{m} \mathrm{m}^{-2} \mathrm{~s}^{-1}$.

A

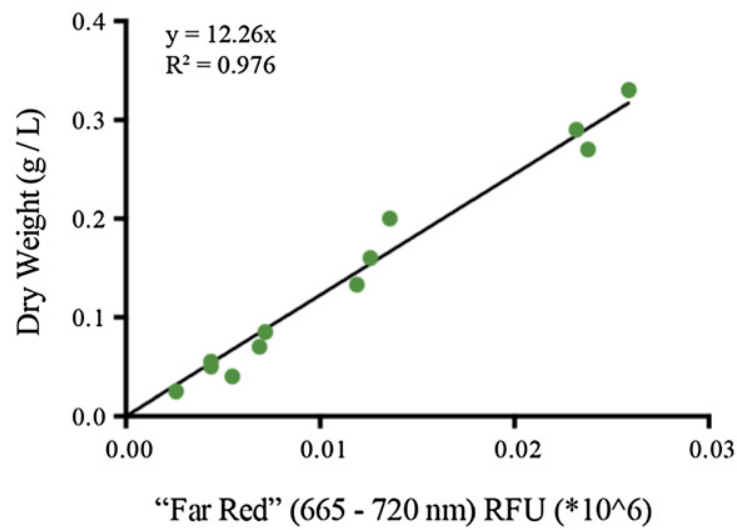

C

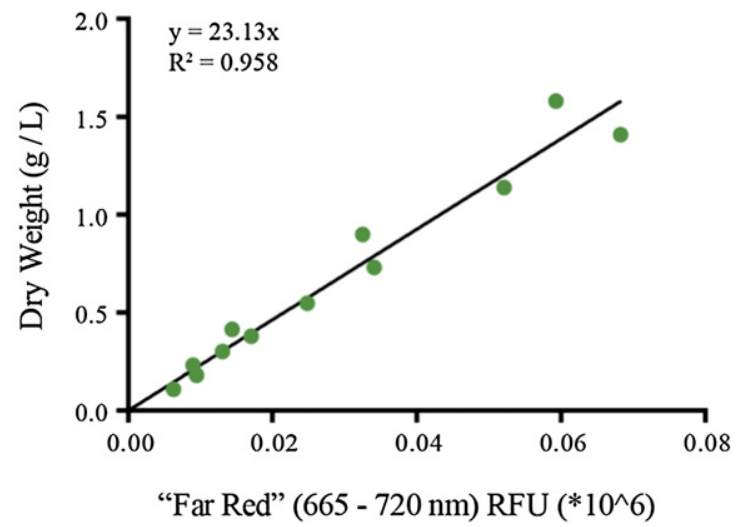

\subsection{Algal fluorescence}

Autofluorescence was measured using the blue excitation and farred emission channels in the Qubit 2.0 raw mode (470 nm excitation, $665-720 \mathrm{~nm}$ emission). For all measurements, at least $300 \mu \mathrm{L}$ of sample was used. Sample tubes were $0.5 \mathrm{~mL}$, thin-wall, clear PCR tubes.

\subsection{Dry weight determination}

Dry weights (DW) were obtained using fritted glass filter assemblies (ChemGlass 1424 assembly) and $4.7 \mathrm{~cm}$ Whatman GF-B glass fiber filter discs. Filters were prewashed five times using $10-15 \mathrm{~mL}$ of DI water and dried overnight in a $105{ }^{\circ} \mathrm{C}$ oven. Filters were allowed to cool in a desiccator before weighing. Weights were taken on an analytical balance readable to $0.1 \mathrm{mg}$. Fluorescence/dry weight correlation curves (Fig. 1) were created for each strain by measuring a series of dilutions created from culture samples.

\subsection{Soluble protein}

$\mathrm{Cr}$ and $\mathrm{Sd}$ were grown in $1 \mathrm{~L}$ flasks containing $250 \mathrm{~mL}$ of TAP media [17] at a temperature of $24{ }^{\circ} \mathrm{C}$ under a constant irradiance of $100 \mu \mathrm{mol} \mathrm{m} \mathrm{m}^{-2} \mathrm{~s}^{-1}$. During logarithmic phase, at a cell density of $1 \cdot 10^{6}$ cells $/ \mathrm{mL}, 250 \mathrm{~mL}$ of culture were spun at a relative centrifugal force (RCF) of $2000 \mathrm{~g}$. The pelleted cells were then resuspended in $15 \mathrm{~mL}$ of tris-buffered saline ( $\mathrm{pH}$ 7.6) and placed on ice. After $10 \mathrm{~min}$
B

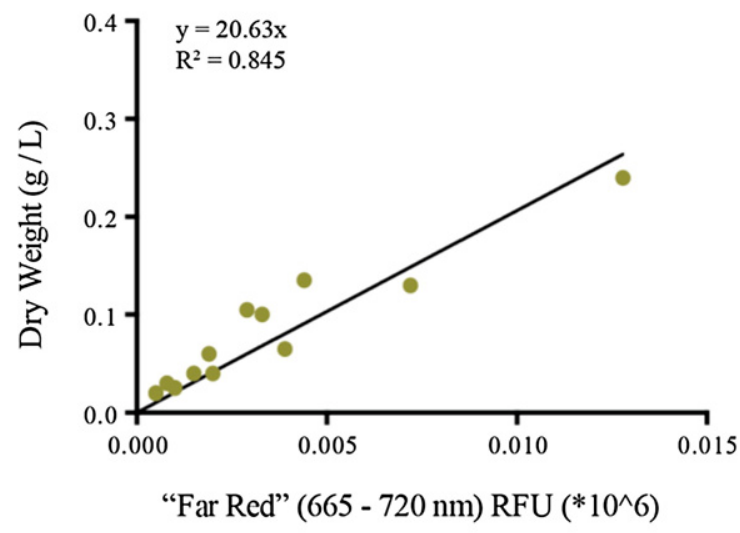

$\mathrm{D}$

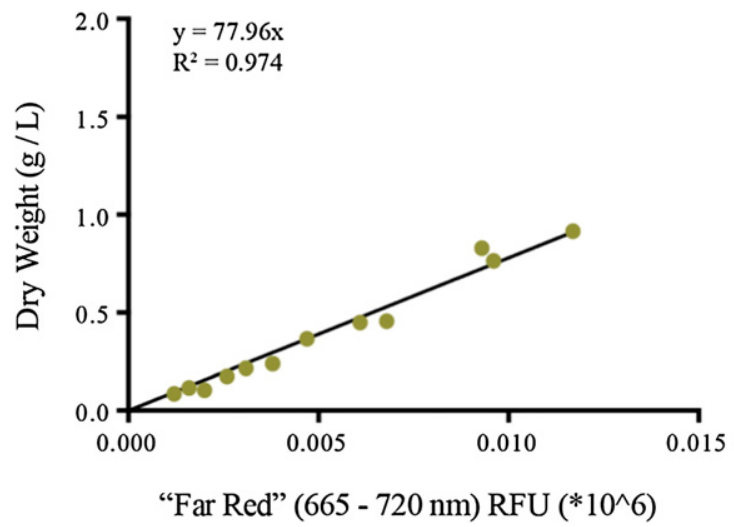

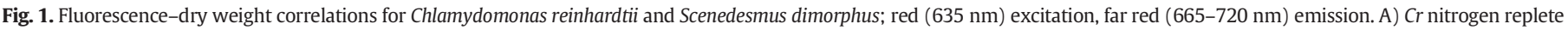

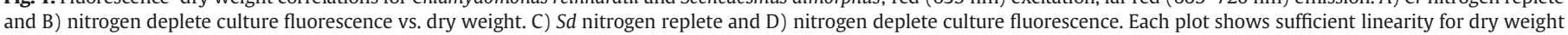
determinations based on rapid fluorescence readings. 
of cooling, cells were sonicated using a Fisher Scientific Sonic Dismembrator Model 500 at 15\% amplitude in two 15 second bursts. Sonicated cells were then incubated on ice for $20 \mathrm{~min}$ and spun in 1.5 eppendorf tubes at 16,000 RCF in an Eppendorf tabletop centrifuge. Instructions for Bio-Rad Bradford and DC (Lowry) assays were followed for the microplate reader versions of the assay, while the Lifetech Qubit assay was performed using the Qubit 2.0 according to manufacturer's instructions. All assays were performed in three technical replicates.

\subsection{Nitrogen starvation experiments}

Growths for nitrogen starvation experiments were conducted using $50 \mathrm{~mL}$ cultures grown in a $\mathrm{CO}_{2}$ chamber at $27.0^{\circ} \mathrm{C}$ under $6500 \mathrm{~K}$ fluorescent lights at a constant irradiance of $95 \mu \mathrm{mol} \mathrm{m}{ }^{-2} \mathrm{~s}^{-1}$. Starter cultures were started from plates by scraping a portion of the plate and resuspending in $1 \mathrm{~mL}$ of the appropriate media before addition to the culture flask. Triplicate nitrogen replete and deplete cultures for nitrogen starvation experiments were inoculated from the same starter culture at a density of $1.0 \cdot 10^{6}$ and $5.0 \cdot 10^{6}$ cells $/ \mathrm{mL}$ for $\mathrm{Cr}$ and $\mathrm{Sd}$ respectively. Cultures were grown for $72 \mathrm{~h}$ before being analyzed for lipid content. All cultures were grown in nitrogen replete or deplete tris-acetate-phosphate (TAP) media.

\subsection{Lipid analysis}

Following $72 \mathrm{~h}$ of growth, $1 \mathrm{~mL}$ samples from each culture were used for lipid estimation via BODIPY fluorescence. $5 \mu \mathrm{L}$ of a $0.1 \mathrm{mM}$ stock solution of BODIPY 495/505 in DMSO was added to each sample to yield a final dye concentration of $0.5 \mu \mathrm{M}$. After $30 \mathrm{~min}$ at room temperature cells were pelleted by centrifugation, washed twice with DI water, and resuspended in fresh media. The samples were then measured using the instrument's blue excitation $(470 \mathrm{~nm})$ and green emission filter $(510-580 \mathrm{~nm})$. A cell count was taken before and after washing. Samples of known concentration were also collected and prepared for GCMS analysis by resuspension in $0.5 \mathrm{~mL}$ of $1 \mathrm{M}$ hydrochloric acid in methanol in Eppendorf tubes. Seven microliters of a $10 \mathrm{mM}$ tridecanoic acid in methanol solution was added to each sample as an internal standard. The methanolic acid samples were incubated at $70{ }^{\circ} \mathrm{C}$ for one hour before being extracted twice with hexane. The hexane extracts of each sample were pooled in GCMS vials for analysis. The run was performed on $1 \mu \mathrm{L}$ of sample with splitless injection. Samples were separated on a $60 \mathrm{~m}$ DB23 Agilent GCMS column using helium as carrier gas and a gradient of $110^{\circ} \mathrm{C}$ to $200^{\circ} \mathrm{C}$ at $15^{\circ} \mathrm{C} / \mathrm{min}$, followed by 20 min at $200{ }^{\circ} \mathrm{C}$.

\section{Results}

\subsection{Algal fluorescence and dry weight determination}

To determine total biomass density, a dry weight-autofluorescence curve was generated for both nitrogen replete and nitrogen deplete cultures of each experimental strain (Fig. 1). The two curves were necessary due to the expected variation in pigment concentration between the two conditions, likely as a result of cannibalization of photosynthetic protein-pigment complexes under nitrogen deplete conditions.

The linear range of algal fluorescence on the instrument was determined to be $0-5 \cdot 10^{6}$ relative fluorescence units (RFU) using excitation/emission wavelengths of $470 / 665-720 \mathrm{~nm}$, and $0-8 \cdot 10^{3}$ RFU when using excitation/emission wavelengths of 635/665-720 nm. Measurements outside of the linear range were easily accomplished by sample dilution. Fluorescence vs. DW plots showed strong linearity for both strains tested (Fig. 1).

\subsection{Soluble protein}

Soluble protein measurements of each strain using both the Bradford and Lowry methods showed excellent agreement with concentrations measured with the fluorometer. Total soluble protein concentration of $\mathrm{Cr}$ was determined to be $2.17 \pm 0.15,2.10 \pm 0.16$, and $2.14 \pm 0.06 \mu \mathrm{g} / \mathrm{mL}$ using the Bradford, Lowry, and Qubit assays respectively. Soluble protein level in $S d$ were determined to be $2.29 \pm$ $0.07,2.09 \pm 0.18,2.24 \pm 0.01 \mu \mathrm{g} / \mathrm{mL}$ using the same assays. These results are averages and standard deviation from three technical replicates diluted to the linear range of each assay. Overall relative standard deviation between assays for both $\mathrm{Cr}$ and $\mathrm{Sd}$ was less than $5 \%$.

\subsection{Lipid content}

Relative lipid content was determined using the fluorescence signal from BODIPY 495/505 stained samples. Measuring lipid via fluorescence provides a relative measure of lipid content, and a rapid comparison of populations of the same strain. For total lipid estimation, relative fluorescence can be correlated to total lipid [18], which is commonly measured via GC/MS. Under nitrogen deplete conditions, both strains showed an accumulation of lipid as measured by relative fluorescence at $72 \mathrm{~h}$ of growth (Fig. 2). This increase correlated to an increase in lipid levels as measured by FAME analysis via GC/MS.

Fluorescence signals were normalized to cell counts post-washing to account for losses during this step, and showed good correlation with lipid levels observed by GC/MS.

\section{Discussion}

\subsection{Algal fluorescence and dry weight determination}

The standard set of LEDs and emission filters on the instrument proved adequate for determination of chlorophyll fluorescence from the two strains of microalgae tested. In-depth studies on the use of chlorophyll autofluorescence as a means of estimating dry weight have been conducted [19], and here we present a basic correlation between the two for both nitrogen replete and deplete conditions. Although convenient and consistent for both strains tested, it is likely that chlorophyll fluorescence vs. dry weight correlation curves would need to be established for both indoor and outdoor conditions due to varying chlorophyll production under different light regimes. However, with the current speed of chlorophyll measurements using the instrument, dry weight measurements would remain the bottleneck. Typically, chlorophyll fluorescence is measured with an excitation wavelength of $440 \mathrm{~nm}$, and emission wavelengths of $660-700 \mathrm{~nm}$. The blue excitation LED (470 nm) coupled with the far-red emission filter (665-720 nm) proved adequate for chlorophyll fluorescence readings over a wide range of culture densities. Again, it is important to note that the slope of the best-fit line varies significantly between strains, and thus fluorescence vs. dry weight correlations must be obtained for each strain and growth condition under investigation. This is likely due to natural variations in pigment composition between various strains of microalgae [20]. Despite this limitation, both excitation wavelengths proved adequate for correlations, allowing for the possibility of dry weight and lipid determination with only two fluorescence readings on a single device.

\subsection{Soluble protein}

Fluorometers have been used previously in the determination of DNA concentration in microalgal cultures [21], and here we demonstrate their additional utility in measuring soluble protein concentration. Comparison of the DC and Bradford assays to the Lifetech fluorescent assay reveals consistent results between the three assays 

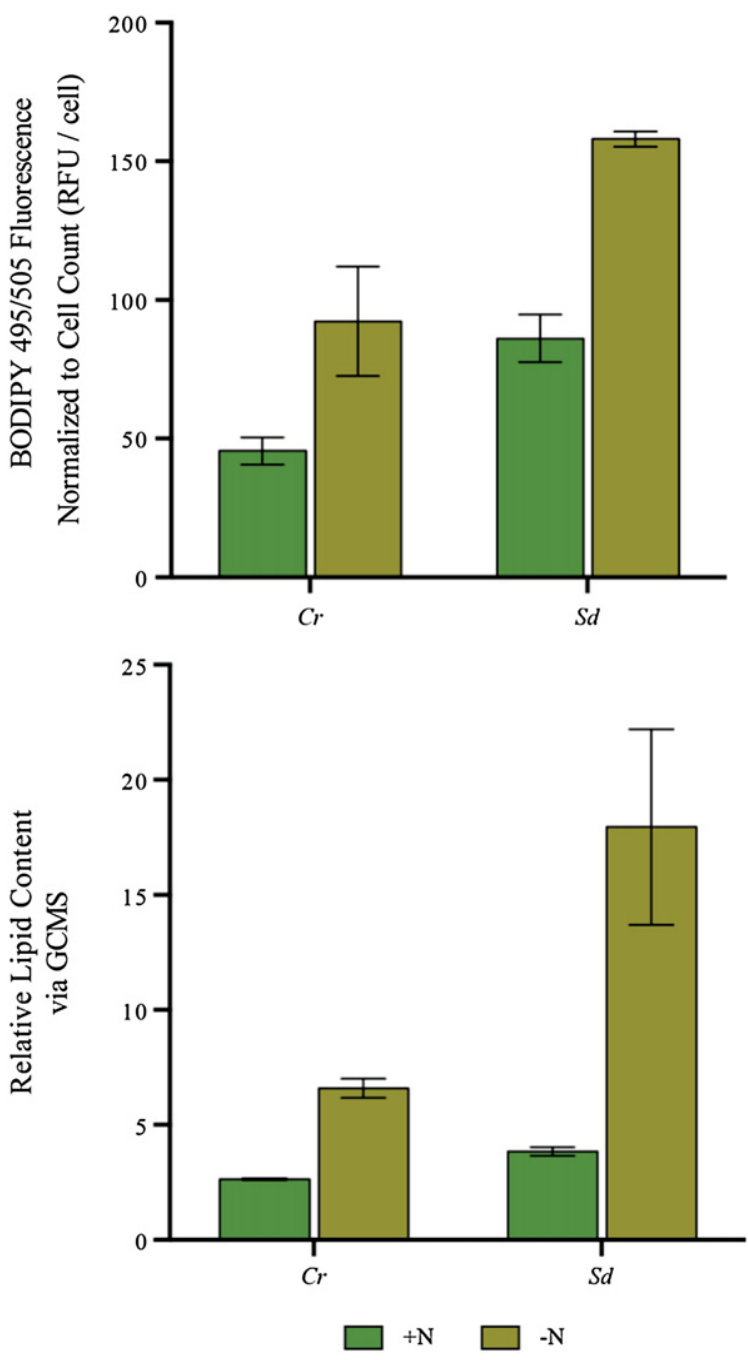

Fig. 2. Total lipid measured via BODIPY 495/505 fluorescence. Both $\mathrm{Cr}$ and $\mathrm{Sd}$ showed a marked increase in lipid, as expected, following $72 \mathrm{~h}$ of growth in nitrogen deplete media. Triplicate cultures were grown and tested for lipid content. For the speed of the assay, good correlation is shown between BODIPY fluorescence and GC/MS data.

despite their use of different measurement methods (absorbance vs. fluorescence) and wavelengths. Samples of algae lysate measured by all three techniques and between the two strains tested yielded values within $1-7 \%$ of each other.

Traditional assays for the measurement of protein content rely on the use of plate readers measuring absorbance from a protein binding dye. While these assays are widely used and reliable, they are not especially well suited to field measurements or transport of the instrumentation. The assay we present is capable of producing results with the same accuracy as traditional Bradford or Lowry protein assays using a portable fluorometer and fluorescent dye. The protein assay reagent interacts with detergent-protein complexes and when bound, fluoresces green in response to excitation at $420 \mathrm{~nm}$. Additionally, Bradford and Lowry are affected by detergents and salts in solution and have a more narrow linear range at 0 to $2 \mathrm{mg} / \mathrm{mL}$ for Bradford and 0 to $0.25 \mathrm{mg} / \mathrm{mL}$ for Lowry compared to 0 to $5 \mathrm{mg} / \mathrm{mL}$ in the fluroscent protein assay. All three assays tested showed highly similar results, demonstrating the usefulness of LED fluorometry in measuring the total soluble protein content of microalgae cultures.

\subsection{Lipid content}

Total lipid is most accurately determined gravimetrically via solvent extraction, but this technique is not well suited to small-scale analysis.
The process involves various drawbacks such as the need for large amounts of biomass, multiple solvent extractions, and relatively long processing times [3]. Faster measurements of lipid content require derivatization of triacylglycerols to fatty-acid esters followed by analysis using gas chromatography/mass spectrometry to provide a quantitative output. GC-MS instruments are typically expensive, although they are well suited to high-throughput, small-scale measurements. Variations can also exist in lipid content measured using GC-MS [22].

Here we have demonstrated the use of an LED fluorometer to obtain a rapid estimation of total lipid, which can then be correlated to traditional GC/MS or gravimetric measurements. The fluorescence signal of the dyes measured in the fluorometer provided a rapidly determined relative measure of lipid content (Fig. 2). This technique is best suited to determining differences in lipid content between two cultures of the same species of algae, due to the variation in baseline signal of different species. The difference in baseline signal in S. dimorphus (UTEX 1237) and C. reinhardtii (CC3491) is likely due to the difference in permeability of the Bodipy dyes between strains. $\mathrm{Cr}$ is a cell wall deficient mutant while $S d$ possesses a tough cell wall which could reduce permeability of the dye [23]. However, using this technique, qualitative comparisons between cultures of the two species showed good correlation to GC/MS measurements. This technique is well suited for obtaining lipid data during different growth phases which could rapidly be compared in order to optimize harvesting and growth processes based on lipid content. The techniques can be adapted to provide a quantitative assessment of lipid content by correlating the fluorescence data to a quantitative technique. Cell counts were a necessary part of the assay to account for strain variability and losses in the washing step, but this could be replaced using the previously discussed estimation of dry cell weight using chlorophyll by relating chlorophyll fluorescence to cell counts. Different species of algae have diverse cell wall types even within families of algae that could potentially lead to variation in BODIPY signal intensity for a given lipid content [24], but with proper controls, the fluorometer provides a rapid measure of total lipid. Lastly, while it may be difficult to compare total lipid levels between strains due to inherent differences in dye penetration, our results demonstrate that lipid accumulation in a single strain under different environmental conditions can be rapidly assayed using the fluorometer.

\section{Conclusion}

As techniques for the monitoring of growth and cell composition of microalgae expand, there will be a push for rapid assays and field instrumentation capable of producing accurate and reliable measurements. We have demonstrated that LED fluorometers can be used as quick and accurate instruments to assess biomass, soluble protein, and lipid content of microalgae cultures. Significant biodiversity of algae makes standardization of assays a challenge. However, the framework set in this study can serve as a foundation to apply these techniques to any strain of microalgae. The methods described here can be used for direct qualitative comparison between cultures of a strain, without the need for more time-consuming methods or expensive instrumentation.

\section{Acknowledgments}

The authors wish to acknowledge the United States Department of Energy (DOE) DE-EE0003373, the California Energy Commission CILMSF 500-10-039, and Thermo Fisher Scientific's Collaborative Research Compacts Program for funding this research.

\section{References}

[1] C. Zhu, Y. Lee, Determination of biomass dry weight of marine microalgae, J. Appl. Phycol. 9 (1997) 189-194. 
[2] E. Barbarino, S.O. Lourenç, An evaluation of methods for extraction and quantification of protein from marine macro-and microalgae, J. Appl. Phycol. 17 (2005) 447-460.

[3] J. Folch, M. Lees, G. Sloane-Stanley, A simple method for the isolation and purification of total lipids from animal tissues, J. Biol. Chem. 226 (1957) 497-509.

[4] F. Lefevre, A. Chalifour, L. Yu, V. Chodavarapu, P. Juneau, R. Izquierdo, Algal fluorescence sensor integrated into a microfluidic chip for water pollutant detection, Lab Chip 12 (2012) 787-793.

[5] J.J. Lamb, J.J. Eaton-Rye, M.F. Hohmann-Marriott, A cost-effective solution for the reliable determination of cell numbers of microorganisms in liquid culture, Curr. Microbiol. (2013) 1-7.

[6] M. Chen, T. Mertiri, T. Holland, A.S. Basu, Optical microplates for high-throughput screening of photosynthesis in lipid-producing algae, Lab Chip 12 (2012) 3870-3874.

[7] G. Ulrich, R. Ziessel, A. Harriman, The chemistry of fluorescent bodipy dyes: versatility unsurpassed, Angew. Chem. Int. Ed. 47 (2008) 1184-1201.

[8] L. Brennan, A. Blanco Fernández, A.S. Mostaert, P. Owende, Enhancement of BODIPY 505/515 lipid fluorescence method for applications in biofuel-directed microalgae production, J. Microbiol. Methods 90 (2012) 137-143.

[9] J. Valenzuela, A. Mazurie, R.P. Carlson, R. Gerlach, K.E. Cooksey, B.M. Peyton, M.W. Fields, Potential role of multiple carbon fixation pathways during lipid accumulation in Phaeodactylum tricornutum, Biotechnol. Biofuels 5 (2012) 40.

[10] T.C. Adarme-Vega, D. Lim, M. Timmins, F. Vernen, Y. Li, P.M. Schenk, Microalgal biofactories: a promising approach towards sustainable omega-3 fatty acid production, Microb. Cell Factories 11 (2012) 96.

[11] B.A. Rasala, M. Muto, P.A. Lee, M. Jager, R.M. Cardoso, C.A. Behnke, P. Kirk, C.A. Hokanson, R. Crea, M. Mendez, Production of therapeutic proteins in algae, analysis of expression of seven human proteins in the chloroplast of Chlamydomonas reinhardtii, Plant Biotechnol. J. 8 (2010) 719-733.

[12] S.S. Merchant, J. Kropat, B. Liu, J. Shaw, J. Warakanont, TAG, You're it! Chlamydomonas as a reference organism for understanding algal triacylglycerol accumulation, Curr. Opin. Biotechnol. 23 (2012) 352-363.
[13] S.P. Mayfield, A.L. Manuell, S. Chen, J. Wu, M. Tran, D. Siefker, M. Muto, J. MarinNavarro, Chlamydomonas reinhardtii chloroplasts as protein factories, Curr. Opin. Biotechnol. 18 (2007) 126-133.

[14] N.G. Schoepp, R.L. Stewart, V. Sun, A.J. Quigley, D. Mendola, S.P. Mayfield, M.D. Burkart, System and method for research-scale outdoor production of microalgae and cyanobacteria, Bioresour. Technol. 166 (2014) 273-281.

[15] S. Guo, X. Zhao, Y. Tang, C. Wan, M.A. Alam, S.-H. Ho, F. Bai, J.-S. Chang, Establishment of an efficient genetic transformation system in Scenedesmus obliquus, J. Biotechnol. 163 (2013) 61-68.

[16] N. Suoeka, Mitotic replication of deoxyribonucleic acid in Chlamydomonas reinhardtii, Proc. Natl. Acad. Sci. U. S. A. 46 (1960) 83-91.

[17] D.S. Gorman, R. Levine, Cytochrome $\mathrm{f}$ and plastocyanin: their sequence in the photosynthetic electron transport chain of Chlamydomonas reinhardi, Proc. Natl. Acad. Sci. U. S. A. 54 (1965) 1665.

[18] T. Govender, L. Ramanna, I. Rawat, F. Bux, BODIPY staining, an alternative to the Nile Red fluorescence method for the evaluation of intracellular lipids in microalgae, Bioresour. Technol. 114 (2012) 507-511.

[19] J. Serôdio, J.M. Da Silva, F. Catarino, Use of in vivo chlorophyll a fluorescence to quantify short-term variations in the productive biomass of intertidal microphytobenthos, Mar. Ecol. Prog. Ser. 218 (2001) 45-61.

[20] N. Hoepffner, S. Sathyendranath, Effect of pigment composition on absorption properties of phytoplankton, Mar. Ecol. Prog. Ser. 73 (1991) l-23.

[21] M. Yilmaz, E.J. Phlips, D. Tillett, Improved methods for the isolation of cyanobacterial DNA from environmental samples, J. Phycol. 45 (2009) 517-521.

[22] I. Khozin-Goldberg, S. Boussiba, Concerns over the reporting of inconsistent data on fatty acid composition for microalgae of the genus Nannochloropsis (Eustigmatophyceae), J. Appl. Phycol. 23 (2011) 933-934.

[23] Z. Tukaj, J. Bohdanowicz, Sensitivity to fuel diesel oil and cell wall structure of some Scenedesmus (Chlorococcales) strains, Acta Soc. Bot. Pol. 64 (1995) 139-147.

[24] H. Takeda, Sugar composition of the cell wall and the taxonomy of chlorella (Chlorophyceae), J. Phycol. 27 (1991) 224-232. 